\title{
Role of IL-1b in NLRP12-associated autoinflammatory disorders and resistance to anti-IL-1 therapy
}

\author{
Isabelle Jeru ${ }^{1 *}$, Véronique Hentgen², Sylvain Normand ${ }^{3}$, Philippe Duquesnoy ${ }^{1}$, Emmanuelle Cochet ${ }^{4}$, \\ Adriana Delwail ${ }^{3}$, Gilles Grateau ${ }^{4}$, Sandrine Marlin ${ }^{4}$, Serge Amselem¹, Jean-Claude Lecron ${ }^{3}$ \\ From 18th Pediatric Rheumatology European Society (PReS) Congress \\ Bruges, Belgium. 14-18 September 2011
}

\section{Background}

A new class of autoinflammatory syndromes called NLRP12-associated disorders (NLRP12AD) has been associated with mutations in NLRP12. Conflicting data on the putative role of NLRP12 in IL-1b signaling have been generated in vitro.

\begin{abstract}
Aim
This prospective study was undertaken to assess the secretion of IL-1b and three IL-1b-induced cytokines (IL-1Ra, IL-6 and TNF-a) in patients' PBMC cultured $e x$ vivo and to evaluate the patients' response to recombinant IL-1 receptor antagonist (IL-1Ra, anakinra), a major drug in the treatment of autoinflammatory disorders.
\end{abstract}

\section{Methods}

Patients' disease manifestations and cytokine measurements were recorded before anakinra treatment was started, during 14 months of therapy, and after discontinuation of anakinra treatment.

\section{Results}

Spontaneous secretion of IL-1b by patients' PBMC was found to be dramatically increased (80 to 175 -fold) compared to controls. Consistently, anakinra initially led to a marked clinical improvement and to a rapid near-normalization of IL-1b secretion. However, a progressive clinical relapse occurred secondarily, associated with an increase in TNF-a secretion, persistent elevated levels of
IL-1Ra and IL-6 and a reactivation of IL-1b secretion. Anakinra was discontinued after 14 months of therapy.

\section{Conclusion}

Our findings provide in vivo evidence of the crucial role of IL-1b in the pathophysiology of NLRP12AD. This is the first time anakinra has been used to treat this disorder. This study provides new insights into the mechanisms underlying resistance to anti-IL-1 therapy observed in few patients with autoinflammatory syndromes. Our data also point to the potential interest of cytokine ex vivo measurements as predictors of response to treatment.

Author details

${ }^{1}$ INSERM, Paris, France. ${ }^{2}$ Centre Hospitalier de Versailles, Versailles, France.

${ }^{3}$ Université de Poitiers, Poitiers, France. ${ }^{4}$ Assistance Publique - Hôpitaux de Paris, Paris, France.

Published: 14 September 2011

doi:10.1186/1546-0096-9-S1-031

Cite this article as: Jeru et al:: Role of IL-1b in NLRP12-associated

autoinflammatory disorders and resistance to anti-IL-1 therapy. Pediatric Rheumatology 2011 9(Suppl 1):O31. 